\title{
Application of Principal Component Analysis to Aircraft Integrated Condition Monitoring and Assessment
}

\author{
Yanming Yang $^{1+}$, Yingjian Liu ${ }^{2}$ and Youbao Ding ${ }^{1}$ \\ ${ }^{1}$ Qingdao Campus, Naval Aeronautical and Astronautical University \\ ${ }^{2}$ Department of Computer Science and Technology, Ocean University of China
}

\begin{abstract}
Principal component analysis (PCA) is a multivariate statistics technique that uses an orthogonal transformation to convert a set of observations of possibly correlated variables into a set of values of linearly uncorrelated variables called principal components. The aircraft integrated condition index is the important parameter to measure the aviation maintenance support capability. It is indispensable to scientifically analyze aircraft condition index data and to make scientific decisions on aviation maintenance to improve maintenance support capability. Aircraft integrated condition involves a large number of indexes, which is more difficult to monitor and evaluate. This paper presents a method of aircraft integrated condition monitoring and evaluation based on principal component analysis model, then gives its mathematic model and algorithm steps in detail. This method integrates multiple indexes by principal component analysis, uses the cumulative variance contribution rate to identify the principal component variables, and turns many index questions into less overall targets. To extract the most important information from the original data eliminates the information redundancy between the samples, and reduces the index dimension, so that the aircraft integrated condition monitoring and evaluation problems are simplified. The practical applications and results analysis show that the PCA-based method is feasible and effective for aircraft integrated condition monitoring and assessment.
\end{abstract}

Keywords: principal component analysis, aircraft integrated condition, monitoring and assessment, eigenvector and eigenvalue

\section{Introduction}

With the rapid development of aviation equipment, there are a lot of monitoring and assessment problems in the aviation maintenance support. To carry out effective monitoring and assessment of aircraft integrated condition, for the promotion of aviation maintenance work targeted, predictive, and promote scientific maintenance of aviation equipment plays a very important role. Aircraft integrated condition index data is becoming more and more complex. Therefore, there is an important significance to develop an effective approach to aircraft integrated condition monitoring and assessment. Principal component analysis (PCA) is a linear dimensionality reduction technique [1,2]. PCA is a technique used to emphasize variation and bring out strong patterns in a dataset. It's often used to make data easy to explore and visualize [3]. PCA method can generalize lower dimensional representation of the original data, in terms of capturing the data direction that has the largest variance [4]. PCA is commonly used as one step in a series of analyses. For a comprehensive introduction to PCA, the reader is referred to [5]. PCA has been widely used to monitor the aircraft integrated condition with multiple variables and evaluate aviation maintenance support ability.

\section{The Mathematical Model of Principal Component Analysis}

Suppose the study object is $n$ samples, $p$ variable data $(n>p)$. We can represent the original data as the following matrix [6]:

\footnotetext{
+ Corresponding author. Tel.: + 0532-51833335.

E-mail address: yymqd@126.com.
} 


$$
\boldsymbol{X}=\left|\begin{array}{cccc}
x_{11} & x_{12} & \cdots & x_{1 p} \\
x_{21} & x_{22} & \cdots & x_{2 p} \\
\vdots & \vdots & & \vdots \\
x_{n 1} & x_{n 2} & \cdots & x_{n p}
\end{array}\right|=\left(\boldsymbol{X}_{1}, \boldsymbol{X}_{2}, \cdots, \boldsymbol{X}_{p}\right)
$$

Linear transformation of $\boldsymbol{X}$ can form a new integrated variable $\boldsymbol{Y}$ :

$$
\boldsymbol{Y}=\boldsymbol{X} \boldsymbol{U}
$$

where $\boldsymbol{Y}=\left(\boldsymbol{Y}_{1}, \boldsymbol{Y}_{2}, \cdots, \boldsymbol{Y}_{p}\right), \boldsymbol{U}=\left(\boldsymbol{u}_{1}, \boldsymbol{u}_{2}, \cdots, \boldsymbol{u}_{p}\right)$.

The $k$-th integrated variable is:

$$
\boldsymbol{Y}_{k}=\boldsymbol{X} \boldsymbol{u}_{k}
$$

where $\boldsymbol{u}_{k}$ is the coefficient of linear transformation.

According to the requirements of the PCA, the following constraints are applied to the linear transformation: (1) $\boldsymbol{Y}_{i}$ is not related to $\boldsymbol{Y}_{j}(i \neq j ; i, j=1,2, \cdots, p)$; (2) $\boldsymbol{Y}_{l}$ is the largest variance in all linear combinations of $\boldsymbol{X}_{1}, \boldsymbol{X}_{2}, \ldots, \boldsymbol{X}_{p}, \boldsymbol{Y}_{2}$ is the largest variance of all linear combinations of $\boldsymbol{X}_{1}, \boldsymbol{X}_{2}, \ldots, \boldsymbol{X}_{p}$ that are not related to $\boldsymbol{Y}_{1}, \boldsymbol{Y}_{2}, \ldots, \boldsymbol{Y}_{p} \ldots, \boldsymbol{Y}_{p}$ is the largest variance of all linear combinations of $\boldsymbol{X}_{1}, \boldsymbol{X}_{2}, \ldots, \boldsymbol{X}_{p}$ that are not related to $\boldsymbol{Y}_{1}, \boldsymbol{Y}_{2}, \ldots, \boldsymbol{Y}_{p}$; (3) $\sum_{i=1}^{p} u_{i k}^{2}=1$, that is $\boldsymbol{u}_{k}^{\prime} \boldsymbol{u}_{k}=1$.

The resulting integrated variable $\boldsymbol{Y}_{l}, \boldsymbol{Y}_{2}, \ldots, \boldsymbol{Y}_{p}$ are the first, second, and $p$-th principal component of the original variable, respectively.

\section{The Algorithm Steps of Principal Component Analysis}

\subsection{Calculate the Correlation Matrix}

To calculate the correlation matrix of the standardized matrix $\boldsymbol{R}$ :

$$
\boldsymbol{R}=\left(r_{i j}\right)_{p \times p}=\frac{\boldsymbol{X}^{\prime} \boldsymbol{X}}{n-1}
$$

where $r_{i j}=\frac{1}{n-1} \sum_{k=1}^{n} x_{k i} x_{k j}, i, j=1,2, \cdots, p$.

\subsection{Calculate the Eigenvectors and Eigenvalues to Determine the Principal Components}

Using Jacobi method to solve the characteristic equation of the correlation coefficient matrix $\boldsymbol{R}$, we obtain the eigenvalues $\lambda_{1}, \lambda_{2}, \ldots, \lambda_{p}\left(\lambda_{1} \geq \lambda_{2} \geq \ldots \geq \lambda_{p}\right)$, and the corresponding eigenvector $\boldsymbol{e}_{i}=\left(e_{i 1}, e_{i 2}, \cdots e_{i p}\right), i=1,2, \cdots, p$.

Then its eigenvalues $\lambda_{i}$ and corresponding eigenvector $\boldsymbol{e}_{i}$ of coefficient matrix $\boldsymbol{R}$, can be also computed respectively as follows:

$$
\begin{gathered}
|\boldsymbol{R}-\lambda \boldsymbol{I}|=0 \\
{\left[\lambda_{i} \boldsymbol{I}-\boldsymbol{R}\right] \boldsymbol{e}_{i}=\mathbf{0}}
\end{gathered}
$$

where $\boldsymbol{R}$ is the Correlation coefficient matrix, $\boldsymbol{I}$ is the $p$-order unit matrix, $\boldsymbol{e}_{i}=\left[\boldsymbol{e}_{i}(1), \boldsymbol{e}_{i}(2), \cdots, \boldsymbol{e}_{i}(n)\right]^{\prime}$.

\subsection{Calculate the Variance Contribution Rate}

The variance contribution rate of the $k$-th principal component $\boldsymbol{Y}_{k}$ :

$$
\alpha_{k}=\lambda_{k} / \sum_{i=1}^{p} \lambda_{i} \quad, i=1,2, \cdots, p
$$

The cumulative variance contribution of the former $m$ principal components $\boldsymbol{Y}_{1}, \boldsymbol{Y}_{2}, \cdots, \boldsymbol{Y}_{m}$ :

$$
\sum_{i=1}^{m} \alpha_{i}=\sum_{i=1}^{m} \lambda_{i} / \sum_{i=1}^{p} \lambda_{i} \quad, m<p
$$


In general, the number of principal components $k$ value is determined by the variance contribution rate $\sum_{i=1}^{m} \alpha_{i} \geq 85 \%$.

\subsection{Comprehensive Evaluation of Principal Component}

First, calculate the linear weighting of each principal component:

$$
\boldsymbol{F}_{i}=\sum_{k=1}^{p} \boldsymbol{F}_{i k} \quad, i=1,2, \cdots, m
$$

where $\boldsymbol{F}_{i k}=\sum_{j=1}^{n} \boldsymbol{e}_{k j} \boldsymbol{X}_{j i}^{\prime} \quad, i=1,2, \cdots, m ; k=1,2, \cdots, p$.

And then $k$ principal components of the weighted sum, that is, the final evaluation value. The weight is the variance contribution rate of each principal component.

$$
\boldsymbol{F}=\sum_{i=1}^{m} \alpha_{i} \boldsymbol{F}_{i} \quad, i=1,2, \cdots, m
$$

where $\alpha_{i}$ is the variance contribution rate of the $i$-th principal component.

\section{An Application Example}

\subsection{Problem Description}

The seven aircraft integrated condition index data of 20 aircrafts in five years: flight sorties, flight time, intact rate, all state intact rate, maintenance grounding rate, failure rate, air failure rate is shown in Table 1. Try to evaluate integrated condition for each aircraft.

Table 1: Aircraft Integrated Condition Index Data in Five Years

\begin{tabular}{|c|c|c|c|c|c|c|c|}
\hline $\begin{array}{c}\text { Aircraft } \\
\text { ID }\end{array}$ & $\begin{array}{c}\text { Flight Sorties } \\
(\mathbf{X 1})\end{array}$ & $\begin{array}{c}\text { Flight Time/h } \\
(\mathbf{X 2})\end{array}$ & $\begin{array}{c}\text { Intact Rate/\% } \\
(\mathbf{X 3})\end{array}$ & $\begin{array}{c}\text { All State } \\
\text { Intact Rate/\% } \\
(\mathbf{X 4})\end{array}$ & $\begin{array}{c}\text { Grounding } \\
\text { Rate/\% } \\
(\mathbf{X 5})\end{array}$ & $\begin{array}{c}\text { Failure } \\
\text { Rate/\% } \\
(\mathbf{X 6})\end{array}$ & $\begin{array}{c}\text { Air Failure } \\
\text { Rate/\% } \\
\text { (X7) }\end{array}$ \\
\hline 1 & 651 & 868.72 & 83.16 & 78.56 & 11.81 & 20.14 & 2.19 \\
\hline 2 & 609 & 857.98 & 89.68 & 85.45 & 7.16 & 22.96 & 3.03 \\
\hline 3 & 618 & 845.86 & 89.14 & 82.12 & 5.91 & 19.15 & 2.60 \\
\hline 4 & 556 & 747.63 & 90.16 & 85.66 & 6.61 & 21.40 & 3.08 \\
\hline 5 & 634 & 848.45 & 92.09 & 87.33 & 4.43 & 20.98 & 2.83 \\
\hline 6 & 537 & 719.60 & 86.01 & 79.68 & 7.78 & 23.76 & 3.20 \\
\hline 7 & 562 & 767.53 & 83.60 & 78.24 & 11.33 & 22.15 & 3.26 \\
\hline 8 & 583 & 805.36 & 88.25 & 80.11 & 6.07 & 20.36 & 2.98 \\
\hline 9 & 616 & 794.78 & 78.18 & 70.05 & 12.66 & 21.89 & 3.27 \\
\hline 10 & 613 & 853.89 & 86.69 & 81.23 & 6.79 & 13.94 & 0.82 \\
\hline 11 & 489 & 662.80 & 85.13 & 80.41 & 10.76 & 17.95 & 3.47 \\
\hline 12 & 660 & 839.35 & 91.86 & 86.35 & 4.52 & 15.73 & 2.50 \\
\hline 13 & 632 & 876.00 & 84.65 & 78.09 & 11.13 & 17.35 & 2.28 \\
\hline 14 & 601 & 821.15 & 86.72 & 81.27 & 9.76 & 18.27 & 1.70 \\
\hline 15 & 642 & 853.71 & 91.56 & 87.59 & 4.16 & 19.56 & 3.16 \\
\hline 16 & 630 & 812.75 & 85.95 & 78.99 & 6.47 & 19.25 & 2.42 \\
\hline 17 & 627 & 851.33 & 86.42 & 82.31 & 8.62 & 17.74 & 2.58 \\
\hline 18 & 538 & 784.10 & 89.33 & 85.01 & 4.90 & 19.39 & 3.32 \\
\hline 19 & 577 & 767.46 & 89.56 & 80.98 & 5.95 & 17.07 & 3.78 \\
\hline 20 & 502 & 724.78 & 92.36 & 86.37 & 3.51 & 19.45 & 2.48 \\
\hline
\end{tabular}

\subsection{Analysis Process}

Calculate the Correlation Coefficient Matrix. According to the correlation between the two index data, calculate the correlation coefficient of the index data. For the same index data, the correlation coefficient is 1 , 
with 1 as the boundary, the diagonal elements take the same value. According to the Eq. (4), we can get the correlation coefficient matrix $\boldsymbol{R}$.

$$
\boldsymbol{R}=\left[\begin{array}{rrrrrr}
1.000 & -0.913 & -0.204 & 0.057 & 0.064 & 0.147 \\
-0.913 & 1.000 & 0.184 & -0.068 & -0.092 & -0.152 \\
-0.204 & 0.184 & 1.000 & 0.517 & -0.283 & -0.280 \\
0.057 & -0.068 & 0.517 & 1.000 & -0.294 & -0.360 \\
0.064 & -0.092 & -0.283 & -0.294 & 1.000 & 0.920 \\
0.147 & -0.152 & -0.280 & -0.360 & 0.920 & 1.000
\end{array}\right]
$$

Calculate the Eigenvectors and Eigenvalues. That is, the eigenvalues and eigenvectors of the correlation matrix $\boldsymbol{R}$ are calculated.

According to the Eq. (5) to calculate the eigenvalue:

$$
\lambda=(2.8288,2.4312,1.0293,0.4230,0.1951,0.0684,0.0242)^{\prime}
$$

According to the Eq. (6) to calculate the corresponding eigenvectors as shown in Table 2.

Table 2: Eigenvectors of the Correlation Matrix

\begin{tabular}{|c|c|c|c|c|c|c|c|}
\hline Variables & PC1 & PC2 & PC3 & PC4 & PC5 & PC6 & PC7 \\
\hline X1 & 0.071 & 0.551 & 0.428 & 0.262 & 0.086 & 0.658 & 0.000 \\
\hline $\mathbf{X 2}$ & 0.134 & 0.560 & 0.379 & -0.002 & -0.114 & -0.715 & 0.001 \\
\hline $\mathbf{X 3}$ & 0.574 & -0.139 & 0.046 & -0.005 & -0.091 & 0.038 & 0.799 \\
\hline $\mathbf{X 4}$ & 0.554 & -0.117 & 0.066 & -0.175 & -0.611 & 0.145 & -0.500 \\
\hline $\mathbf{X 5}$ & -0.546 & 0.130 & -0.040 & -0.063 & -0.750 & 0.099 & 0.327 \\
\hline $\mathbf{X 6}$ & -0.185 & -0.323 & 0.693 & -0.601 & 0.120 & 0.063 & 0.044 \\
\hline $\mathbf{X 7}$ & -0.088 & -0.478 & 0.430 & 0.732 & -0.144 & -0.143 & -0.050 \\
\hline
\end{tabular}

Determine the Principal Components. In order to determine the number of principal components, the cumulative variance contribution rate should be calculated first.

According to the Eq. (7) and Eq. (8) to calculate the variance contribution rate and cumulative variance contribution as shown in Table 3.

Table 3: Total Variance Explained

\begin{tabular}{|c|c|c|c|c|c|c|}
\hline \multirow{2}{*}{ Component } & \multicolumn{2}{|c|}{ Initial Eigenvalue } & \multicolumn{4}{|c|}{ Extraction Sums of Squared Loadings } \\
\cline { 2 - 7 } & Total & Variance/\% & Cumulative/\% & Total & Variance/\% & Cumulative/\% \\
\hline 1 & 2.829 & 40.411 & 40.411 & 2.829 & 40.411 & 40.411 \\
\hline 2 & 2.431 & 34.731 & 75.142 & 2.431 & 34.731 & 75.142 \\
\hline 3 & 1.029 & 14.705 & 89.847 & 1.029 & 14.705 & 89.847 \\
\hline 4 & 0.423 & 6.043 & 95.890 & & & \\
\hline 5 & 0.195 & 2.787 & 98.677 & & & \\
\hline 6 & 0.068 & 0.978 & 99.655 & & & \\
\hline 7 & 0.024 & 0.345 & 100.000 & & & \\
\hline
\end{tabular}

It can be seen from Table 3 that the first three eigenvalues are greater than 1 and the cumulative contribution rate is over $85 \%(89.847 \%)$, which indicates that the information reflected in the six aircraft maintenance index data can be reflected by three principal components, which explain $89.847 \%$ of total variation of variables in PCA.

After conducting PCA, we found that the first three PCs explained $89.847 \%$ of the process variation. Thus, we only used the first three PCs to obtain the variables. Therefore, the expression of the principal component can be calculated according to Eq. (9) and Table 2:

$$
\begin{aligned}
& P C 1=0.071 \boldsymbol{X}_{1}+0.134 \boldsymbol{X}_{2}+0.574 \boldsymbol{X}_{3}+0.554 \boldsymbol{X}_{4}-0.546 \boldsymbol{X}_{5}-0.185 \boldsymbol{X}_{6}-0.088 X_{7} \\
& P C 2=0.551 \boldsymbol{X}_{1}+0.560 \boldsymbol{X}_{2}-0.139 \boldsymbol{X}_{3}-0.117 \boldsymbol{X}_{4}+0.130 \boldsymbol{X}_{5}-0.323 \boldsymbol{X}_{6}-0.478 X_{7} \\
& P C 3=0.428 \boldsymbol{X}_{1}+0.379 \boldsymbol{X}_{2}+0.046 \boldsymbol{X}_{3}+0.066 \boldsymbol{X}_{4}-0.040 \boldsymbol{X}_{5}+0.693 \boldsymbol{X}_{6}+0.430 X_{7}
\end{aligned}
$$




\subsection{Result Analysis}

From the analysis of Fig.1 that: The first principal component includes intact rate (X3), all state intact rate (X4) and grounding rate (X5); the second principal component includes flight sorties (X1) and flight time (X2); the third principal component includes failure rate (X6) and air failure rate (X7).

The analysis shows that the first principal component is expressed as the maintenance support capability, the second principal component is expressed as the strength of the aircraft, and the third principal component is expressed as the inherent reliability of the aircraft.

In order to visually display the assessment result, the distribution state of the principal component score may be represented by a score plot. The main component of the eigenvalue is the coordinate axis, which shows the main component score state, as shown in Fig.2. It can be seen from the figure, for the first principal component, the more the point on the right side, indicating the greater the aircraft maintenance support capacity; for the second main component, the upper point, indicating the greater use of aircraft strength.

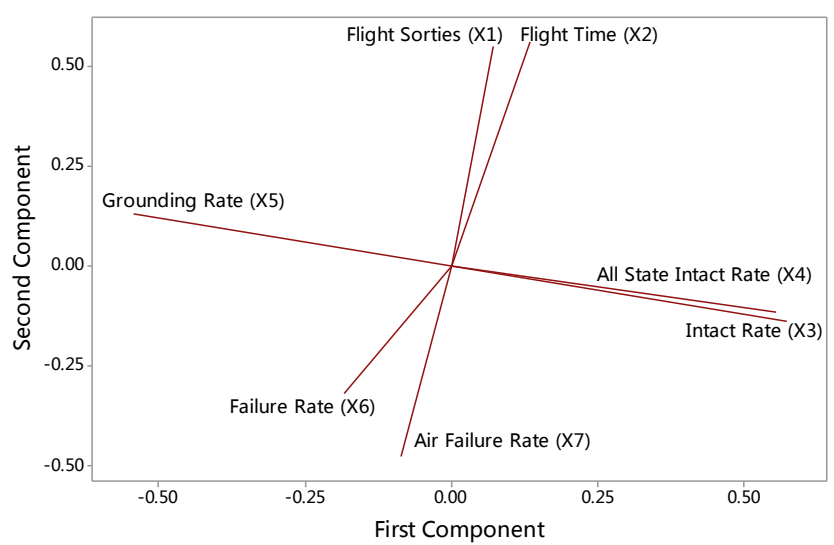

Fig. 1: Loading plot of aircraft integrated condition.

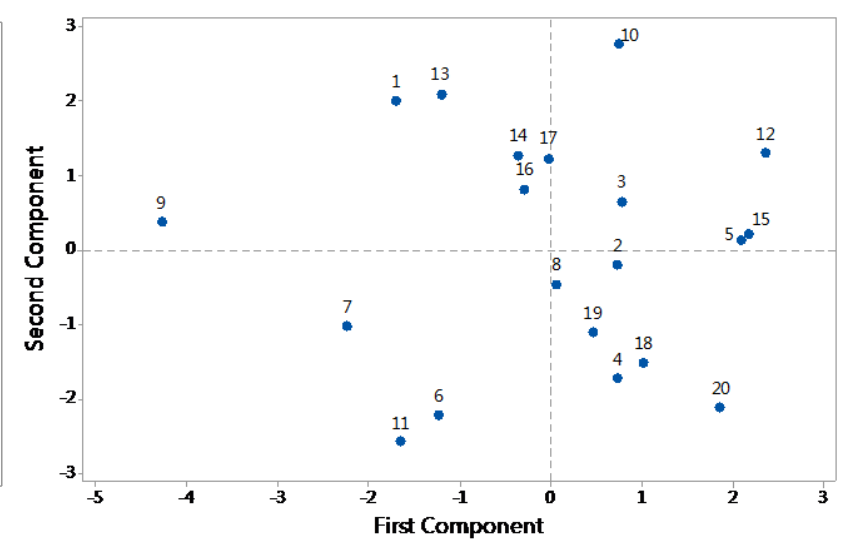

Fig. 2: Score plot aircraft integrated condition.

\section{Conclusion}

Aiming at the aircraft integrated condition monitoring and assessment, an effective approach based on PCA is put forward. Based on the principle of principal component analysis, the mathematical model and algorithm steps are given in detail. In order to improve the operability of the method, taking the aircraft integrated condition index data as an example, this paper discusses how to use the PCA-based method to monitor and evaluate the aircraft integrated condition. And the visual analysis results are presented graphically. The results show that the method proposed in this paper is an effective monitoring and assessment approach to aircraft integrated condition. The method can also be applied to other multivariate evaluation issues in the field of aeronautical equipment maintenance support.

\section{References}

[1] Jolliffe, I. T., and J. Cadima. "Principal component analysis: a review and recent developments. " Philosophical Transactions of the Royal Society A Mathematical Physical \& Engineering Sciences 374.2065(2016):20150202.

[2] Shen, Dan, H. Shen, and J. S. Marron. "A general framework for consistency of principal component analysis." Journal of Machine Learning Research 17.1(2016):5218-5251.

[3] Behdad, Mohammad, et al. "On principal component analysis for high-dimensional XCSR." Evolutionary Intelligence 5.2(2012):129-138.

[4] Wang, Rixin, et al. "Fault detection of flywheel system based on clustering and principal component analysis." Chinese Journal of Aeronautics 28.6(2015):1676-1688.

[5] Abdi. H., \& Williams, L.J. (2010). "Principal component analysis". Wiley Interdisciplinary Reviews: Computational Statistics. 2 (4): 433-459.

[6] Zhengshun, and Kangling. "Online process monitoring for complex systems with dynamic weighted principal component analysis." Chinese Journal of Chemical Engineering 24.6(2016):775-786. 